\title{
Overexpression of the Barley Nicotianamine Synthase Gene HvNAS1 Increases Iron and Zinc Concentrations in Rice Grains
}

\author{
Hiroshi Masuda • Kanako Usuda • Takanori Kobayashi • Yasuhiro Ishimaru • \\ Yusuke Kakei • Michiko Takahashi • Kyoko Higuchi • Hiromi Nakanishi • \\ Satoshi Mori • Naoko K. Nishizawa
}

Received: 27 April 2009 / Accepted: 8 September 2009/Published online: 30 September 2009

(C) The Author(s) 2009. This article is published with open access at Springerlink.com

\begin{abstract}
In humans, iron (Fe) and zinc ( $\mathrm{Zn})$ deficiencies result in major worldwide health problems. Transgenic technologies to produce $\mathrm{Fe}$ - and $\mathrm{Zn}$-biofortified rice varieties offer a promising potential solution. Nicotianamine, the precursor of phytosiderophores, chelates $\mathrm{Fe}^{2+}$ and $\mathrm{Zn}^{2+}$ and plays an important role in transporting these metals to both vegetative and reproductive organs within the plant. Our objective was to increase $\mathrm{Fe}$ and $\mathrm{Zn}$ contents in rice grains by overexpressing the barley nicotianamine synthase gene $H v N A S 1$. HvNASl-overexpressing transgenic
\end{abstract}

H. Masuda $\cdot$ K. Usuda $\cdot$ T. Kobayashi $\cdot$ Y. Ishimaru $\cdot$ Y. Kakei $\cdot$

M. Takahashi $\cdot$ N. K. Nishizawa $(\triangle)$

Department of Global Agricultural Sciences,

Graduate School of Agricultural and Life Sciences,

The University of Tokyo,

1-1-1 Yayoi,

Bunkyo-ku, Tokyo 113-8657, Japan

e-mail: annaoko@mail.ecc.u-tokyo.ac.jp

K. Higuchi

Department of Applied Biology and Chemistry,

Tokyo University of Agriculture,

1-1-1 Sakuraoka,

Setagaya-ku, Tokyo 156-8502, Japan

H. Nakanishi $\cdot$ S. Mori

Department of Applied Biological Chemistry,

Graduate School of Agricultural and Life Sciences,

The University of Tokyo,

1-1-1 Yayoi,

Bunkyo-ku, Tokyo 113-8657, Japan

Present Address:

M. Takahashi

Faculty of Agriculture, Utsunomiya University,

350 Minemachi,

Utsunomiya-shi 321-8505, Tochigi, Japan rice showed increased $H v N A S 1$ expression and subsequent increases in endogenous nicotianamine and phytosiderophore content in shoots, roots, and seeds. Fe and $\mathrm{Zn}$ concentrations in polished $T_{1}$ seeds from transgenic plants increased more than three and twofold, respectively; $\mathrm{Fe}$ and $\mathrm{Zn}$ concentrations also increased in both polished and brown $\mathrm{T}_{2}$ seeds. These results suggest that the overproduction of nicotianamine enhances the translocation of $\mathrm{Fe}$ and $\mathrm{Zn}$ into rice grains.

Keywords Nicotianamine $\cdot H v N A S 1 \cdot$ Iron · Zinc .

Transgenic rice $\cdot$ Biofortification

\section{Introduction}

Iron $(\mathrm{Fe})$ and zinc $(\mathrm{Zn})$ are essential micronutrients in both plants and animals. Shortages in $\mathrm{Fe}$ and $\mathrm{Zn}$ are among the most prevalent human micronutrient deficiencies in the world, each affecting an estimated one third of the world's population (Sandstead 1991; Stoltzfus and Dreyfuss 1998) and resulting, separately, in 0.8 million deaths per year worldwide (WHO 2002). Fe and $\mathrm{Zn}$ deficiencies are the sixth and fifth highest health risk factors, respectively, in developing countries with high mortality rates (WHO 2002). Several approaches have been proposed to decrease the prevalence of micronutrient deficiencies. Biofortification (i.e., breeding micronutrient-fortified crops) is particularly advantageous for people living in rural areas because it does not require specific processing techniques after harvest or specialized infrastructure (Lucca et al. 2006). In addition, transgenic techniques offer the possibility of producing micronutrient-fortified rice varieties.

Transgenic $\mathrm{Fe}$ fortification has been attempted using ferritin, a protein that stores approximately $4,500 \mathrm{Fe}$ atoms 
in a complex. Goto et al. (1999) generated transgenic rice plants that expressed soybean ferritin in the endosperm using the endosperm-specific rice GluB1 promoter; the transformants showed $\mathrm{Fe}$ accumulation in brown rice seeds. However, further enhancement of ferritin expression did not produce further increases in seed $\mathrm{Fe}$ content $(\mathrm{Qu}$ et al. 2005). In addition to increased $\mathrm{Fe}$ storage in seeds, enhanced Fe uptake from the soil and translocation within the plant are thought to be required to further improve $\mathrm{Fe}$ fortification in seeds.

Nicotianamine (NA), a chelator of metal cations such as $\mathrm{Fe}(\mathrm{II})$ and $\mathrm{Zn}(\mathrm{II})$, is biosynthesized from S-adenosyl methionine via NA synthase (NAS; Higuchi et al. 1994). All higher plants synthesize and utilize NA for the internal transport of Fe and other metals (Hell and Stephan 2003; Takahashi et al. 2003). Indeed, the NA-defective tomato mutant chloronerva (Rudolph et al. 1985) presents a phenotype indicative of Fe deficiency (Pich and Scholz 1996; Stephan et al. 1996). Takahashi et al. (2003) produced NA-deficient transgenic tobacco plants by constitutively expressing the barley NA aminotransferase (NAAT) gene, HvNAAT-A. The transformants showed young leaves with interveinal chlorosis, and as a result of disrupted internal metal transport, $\mathrm{Fe}$ and $\mathrm{Zn}$ concentrations in the leaves and flowers decreased. Conversely, overexpressing the barley NAS gene, $H v N A S 1$, led to increased $\mathrm{Fe}$ and $\mathrm{Zn}$ concentrations in the leaves, flowers, and seeds of tobacco plants (Takahashi et al. 2003). Similarly, in Arabidopsis AtNAS quadruple mutant, Fe concentration in seeds was found to decrease (Klatte et al. 2009). These reports suggest that NA plays an essential role for $\mathrm{Fe}$ translocation to seeds.

In addition, Koike et al. (2004) identified the rice NA$\mathrm{Fe}(\mathrm{II})$ transporter gene OsYSL2, which is preferentially expressed in leaf phloem cells and the vascular bundles of flowers and developing seeds, suggesting a role in internal Fe transport.

In graminaceous plants, NA is the precursor of mugineic acid family phytosiderophores (MAs), which are natural $\mathrm{Fe}(\mathrm{III})$ chelators used in $\mathrm{Fe}$ acquisition from the rhizosphere (Mihashi and Mori 1989; Takagi 1976). Under Fe-deficient conditions, graminaceous plants synthesize and secrete MAs into the rhizosphere where they form Fe(III)-MAs complexes that are absorbed into the root via YS1-like transporters (Curie et al. 2001). Rice produces 2'-deoxymugineic acid (DMA), which chelates Fe(III) and contributes to $\mathrm{Fe}$ uptake from the soil and internal transport (Kobayashi et al. 2008). Therefore, increased NA concentrations would also be expected to increase DMA concentrations in rice plants, with subsequent improvements in $\mathrm{Fe}$ uptake and transport.

We hypothesized that the overexpression of the NAS gene in rice would enhance the synthesis of NA and
DMA, and thus increase $\mathrm{Fe}$ and $\mathrm{Zn}$ concentrations in seeds. Higuchi et al. (2001a) introduced the HvNAS1 gene into rice under the control of the $35 \mathrm{~S}$ promoter. The resultant transgenic lines produced two to threefold greater concentrations of NA compared to non-transgenic lines. DMA production and secretion also increased under Fe-sufficient conditions. In this paper, we describe new transgenic rice lines that overexpress the HvNAS1 gene under the control of the rice Actin1 promoter. Using these lines and previously generated $35 S-H v N A S 1$ transformants (Higuchi et al. 2001a), we analyzed HvNAS1 gene expression, endogenous NA and DMA concentrations, and $\mathrm{Fe}$ and $\mathrm{Zn}$ contents in seeds. NA concentrations in the shoots, roots, and seeds of transgenic rice, and $\mathrm{Fe}$ and $\mathrm{Zn}$ concentrations in polished $T_{1}$ rice seeds, increased compared to non-transgenic plants. $\mathrm{Fe}$ and $\mathrm{Zn}$ concentrations also increased in polished and brown $\mathrm{T}_{2}$ seeds in proportion to the expression of the $H v N A S 1$ transgene in $\mathrm{T}_{1}$ plants.

\section{Results}

$\mathrm{Fe}$ and $\mathrm{Zn}$ concentrations in polished $\mathrm{T}_{1}$ seeds from OsActin1-HvNAS1 lines

To determine whether enhanced $N A S$ expression increases $\mathrm{Fe}$ and $\mathrm{Zn}$ concentrations in rice seeds, OsActin1-HvNAS1 (AN) transgenic lines were produced. After harvest from soil culture with slow-release fertilizer, polished and brown rice seeds from both non-transgenic rice (NT) and $12 \mathrm{AN}$ lines were subjected to metal concentration analysis. Fe and $\mathrm{Zn}$ concentrations increased markedly in polished $\mathrm{T}_{1}$ transgenic rice seeds compared to NT seeds (Fig. 1). In line $\mathrm{AN} 1, \mathrm{Fe}$ and $\mathrm{Zn}$ concentrations in polished seeds increased 4.5- and 2.5-fold, respectively, compared to the NT line. Fe and Zn concentrations also increased in brown rice seeds, although to a lesser degree (data not shown). Among 12 AN lines, we selected lines AN1, AN2, and AN3, which showed the highest $\mathrm{Fe}$ and $\mathrm{Zn}$ concentrations in polished rice seeds, for further analysis.

\section{Transgene expression in $H v N A S 1$ overexpression lines}

Three AN lines (AN1, AN2, and AN3) and the NT line were grown hydroponically under Fe-sufficient conditions. A previously generated transgenic line carrying $35 \mathrm{~S}-\mathrm{HvNAS1}$ (35S; Higuchi et al. 2001a) was also grown under the same conditions. HvNAS1 transgene expression in shoots was detected via Northern blot analysis (Fig. 2). HvNAS1 expression was induced in the $35 \mathrm{~S}$ line and three AN lines in Fe-sufficient shoots. The strongest transgene expression was observed in the $35 \mathrm{~S}$ and AN1 lines. 


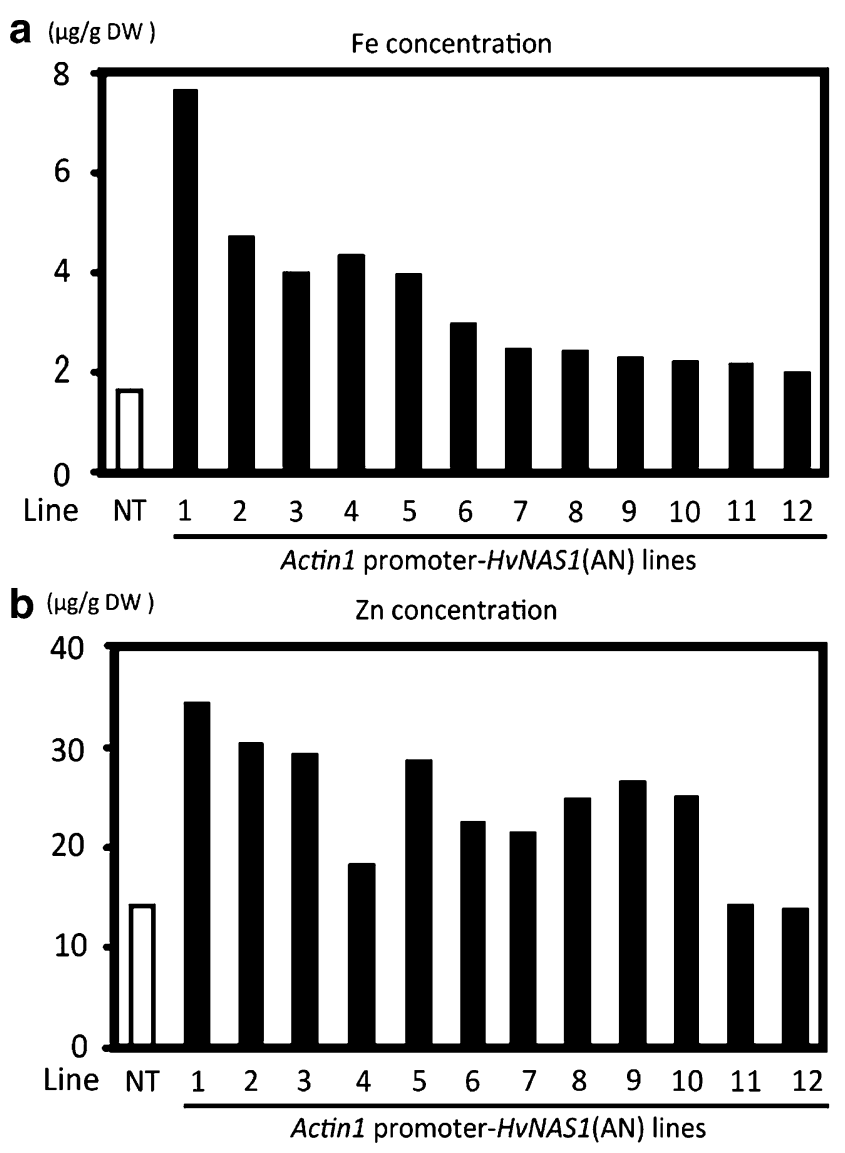

Fig. $1 \mathrm{Fe}$ and $\mathrm{Zn}$ concentrations in polished $\mathrm{T}_{1}$ seeds. Ten well-polished seeds from main tillers were subjected to metal concentration analysis. a $\mathrm{Fe}$ concentration in polished $\mathrm{T}_{1}$ seeds. $\mathbf{b} \mathrm{Zn}$ concentration in polished $\mathrm{T}_{1}$ seeds. NT (white bar), non-transgenic line; numbers (black bars), OsActin1-HvNAS1 (AN) lines.

\section{Endogenous NA and DMA concentrations in $T_{1}$ plants}

Endogenous NA and DMA concentrations in AN, 35S, and NT lines were measured via high-performance liquid chromatography (HPLC; Fig. 3). In the shoots and roots,

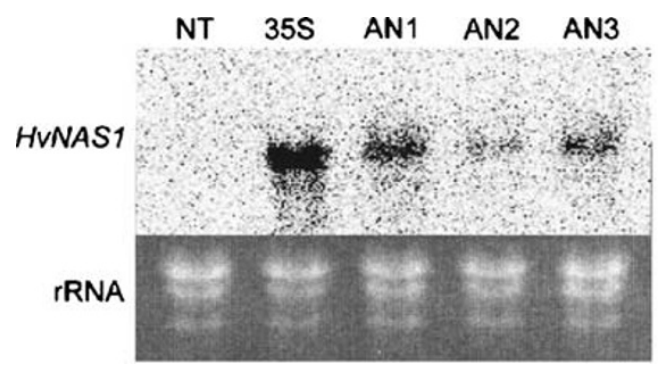

Fig. 2 Northern blot analysis of $H v N A S 1$ gene expression in transgenic lines. The HvNASl ORF was used as a probe. Total RNA was extracted from the shoots of 44-day-old plants grown hydroponically under Fe-sufficient conditions, and $20 \mu \mathrm{g}$ of total RNA was loaded into each lane. Ethidium bromide staining was used to ensure equal loading of rRNA. $N T$ non-transgenic line; $35 S, 35 S-H v N A S 1$ transgenic line; $A N 1$, $A N 2$, and AN3, OsActin1-HvNAS1 transgenic lines.
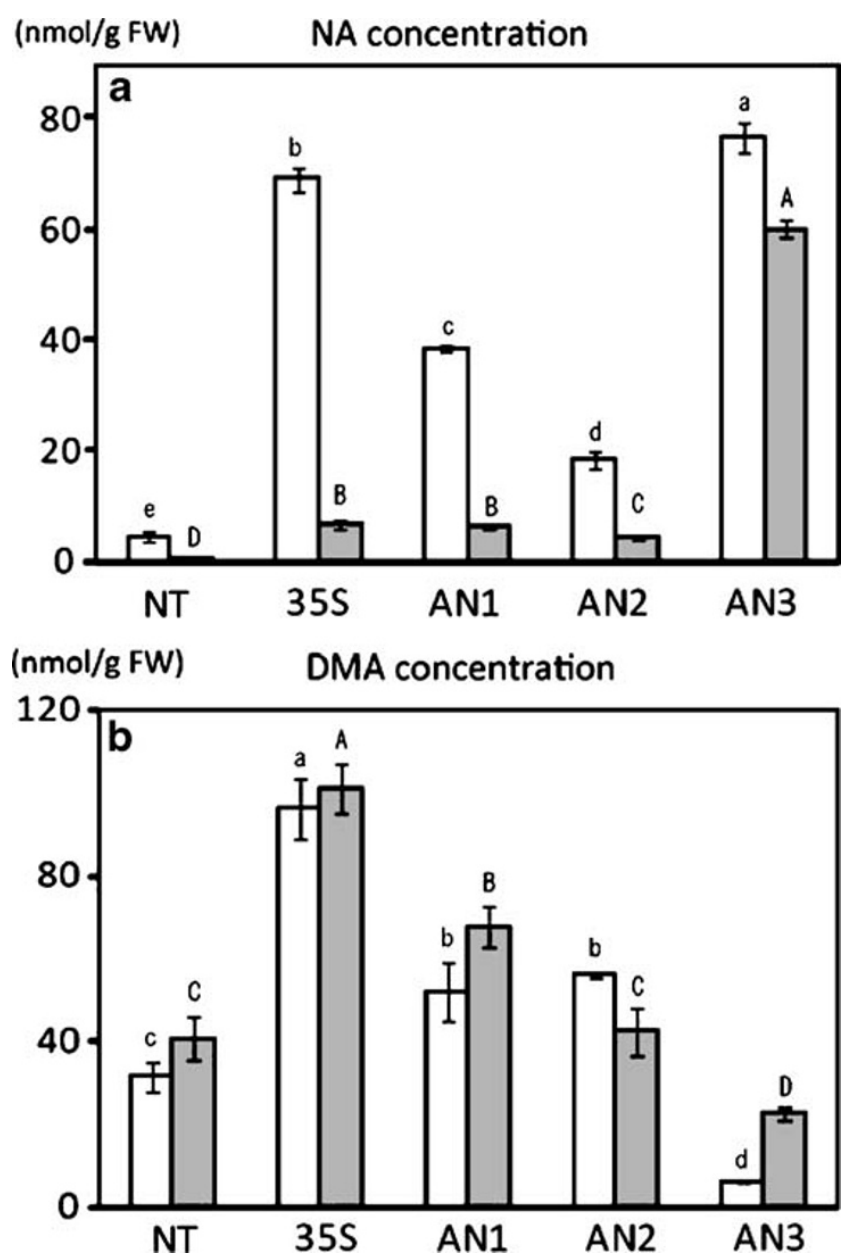

Fig. 3 Endogenous $\mathrm{NA}$ and DMA concentrations in $\mathrm{T}_{1}$ plants determined via high-performance liquid chromatography (HPLC). a NA concentrations. b DMA concentrations. NA and DMA were extracted from 44-day-old plants grown hydroponically under Fe-sufficient conditions. The data represent the mean \pm standard error of three measurements for each sample. White bars, shoot concentration; gray bars, root concentration; NT non-transgenic line; $35 \mathrm{~S}, 35 \mathrm{~S}$ HvNAS1 transgenic line; AN1, AN2, and AN3, OsActin1-HvNAS1 transgenic lines. Distinct letters above the bars indicate significant differences $(p<0.05)$.

endogenous NA concentrations were markedly higher in the $35 \mathrm{~S}, \mathrm{AN} 1, \mathrm{AN} 2$, and $\mathrm{AN} 3$ lines in $\mathrm{T}_{1}$ plants compared to the NT line (Fig. 3a). The most marked increase (approximately 15-fold) was observed in shoots collected from lines 35S and AN3 (Fig. 3a). DMA concentrations were also higher in shoots and roots collected from the 35S, AN1, and AN2 lines compared to the NT line (Fig. 3b).

$\mathrm{Fe}$ and $\mathrm{Zn}$ contents in $\mathrm{T}_{2}$ seeds

To determine whether $\mathrm{Fe}$ and $\mathrm{Zn}$ accumulated in $\mathrm{T}_{2}$ seeds, we cultivated $H v N A S 1$-overexpressing lines in two separate experimental designs. In the first experiment, three AN 
lines (AN1, AN2, and AN3; $\mathrm{T}_{1}$ generation), the 35S line, and NT plants were grown in small pots fertilized with nutrient solution alone. In polished $\mathrm{T}_{2}$ seeds collected from the $\mathrm{AN} 1$ and 35S lines, $\mathrm{Fe}$ and $\mathrm{Zn}$ concentrations increased significantly compared to NT plants (Fig. 4a, b). Fe concentrations in polished rice seeds collected from the 35S and AN1 lines increased 2.3- and 1.6-fold, respectively
(Fig. 4a). Zn concentrations in polished rice seeds collected from the 35S, AN1, and AN3 lines increased 1.5-, 1.2-, and 1.3-fold, respectively, compared to NT plants (Fig. 4b). Fe and $\mathrm{Zn}$ concentrations also increased in brown rice seeds collected from the 35S, AN1, and AN3 lines (Fig. 4c, d). Fe concentrations increased only slightly in rice bran collected from $35 \mathrm{~S}$ and AN3 plants (Fig. 4e), and Zn concentrations
Fig. $4 \mathrm{Fe}$ and $\mathrm{Zn}$ concentrations in $T_{2}$ seeds obtained from plants cultured in small pots fertilized with nutrient solution. $T_{2}$ seeds from the main tillers of NT, $35 \mathrm{~S}$ HvNAS1 (35S), and OsActin1HvNAS1 (AN1, AN2, and AN3) lines were harvested and subjected to metal concentration analysis. a Fe concentration in polished rice seeds. b $\mathrm{Zn}$ concentration in polished rice seeds. c Fe concentration in brown rice seeds. $\mathbf{d} \mathrm{Zn}$ concentration in brown rice seeds. e Fe concentration in bran. f $\mathrm{Zn}$ concentration in bran. $\mathbf{g} \mathrm{Fe}$ concentration in hulls. $\mathbf{h ~} \mathrm{Zn}$ concentration in hulls. The data represent the mean \pm standard error of six independent pots. Distinct letters above the bars indicate significant differences $(p<0.05)$.
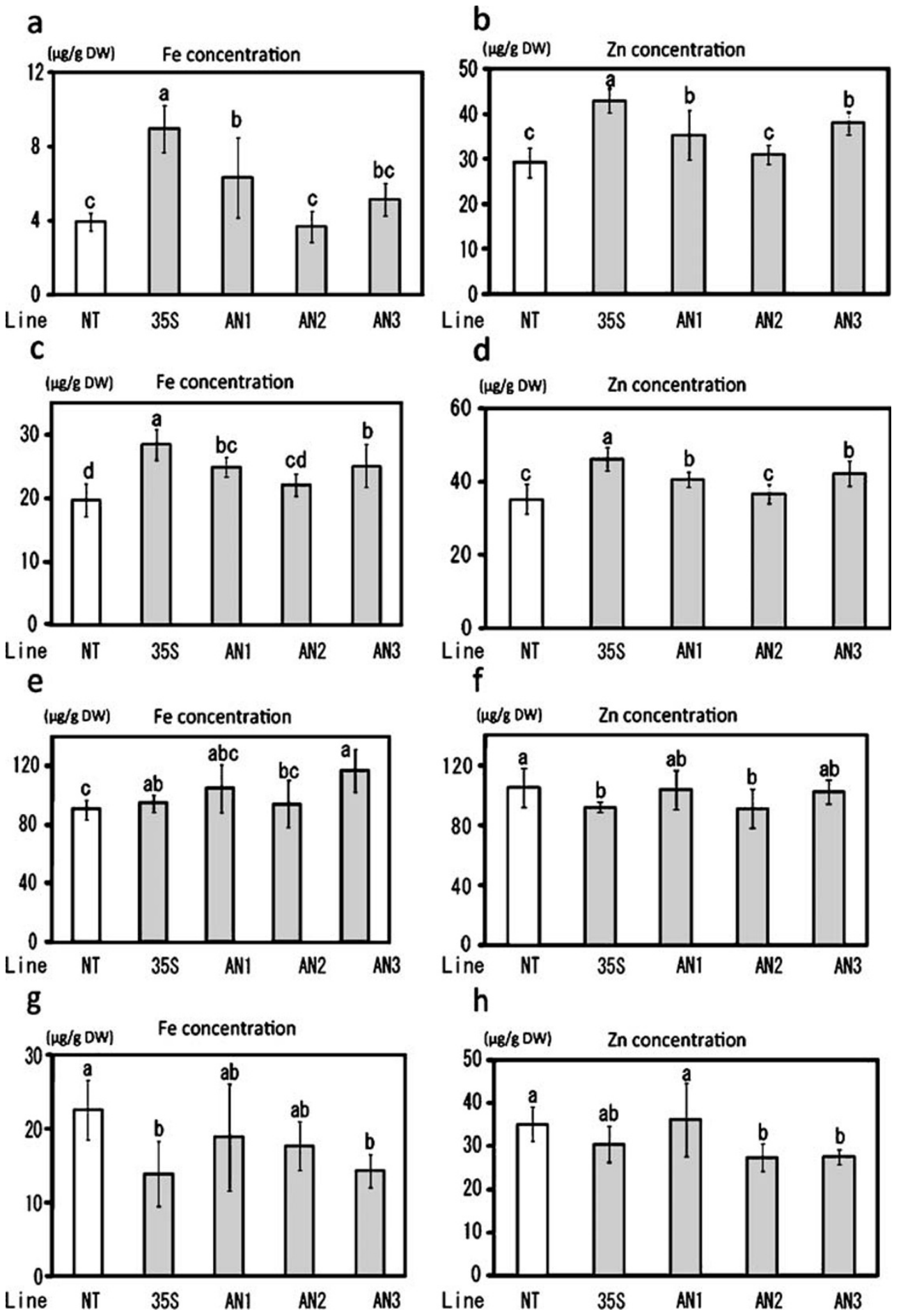
did not increase (Fig. 4f). In rice hulls, $\mathrm{Fe}$ and $\mathrm{Zn}$ concentrations decreased in the $35 \mathrm{~S}$ and $\mathrm{AN}$ lines (Fig. 4g, h). Fe and Zn contents per seed were calculated using the metal concentration data from $\mathrm{T}_{2}$ seeds (Fig. 5a, b). Fe content per seed for polished, brown, and full grains increased in the 35S, AN1, and AN3 lines, whereas Fe content decreased in hulls per seed in the $35 \mathrm{~S}$, AN1, and AN3 lines. $\mathrm{Zn}$ contents showed a similar distribution among the $35 \mathrm{~S}$ and AN3 lines.

NA and DMA contents in $\mathrm{T}_{2}$ seeds

Next, endogenous NA and DMA concentrations in $\mathrm{T}_{2}$ brown rice seeds of $35 \mathrm{~S}, \mathrm{AN} 1, \mathrm{AN} 3$, and $\mathrm{NT}$ lines were measured using liquid chromatography/electrospray ionization time-of-flight mass spectrometry (LC/ESITOF-MS). NA concentrations in $\mathrm{T}_{2}$ seeds of $35 \mathrm{~S}$, AN1, and AN3 lines increased 10.6-, 5.1-, and 4.7-fold,
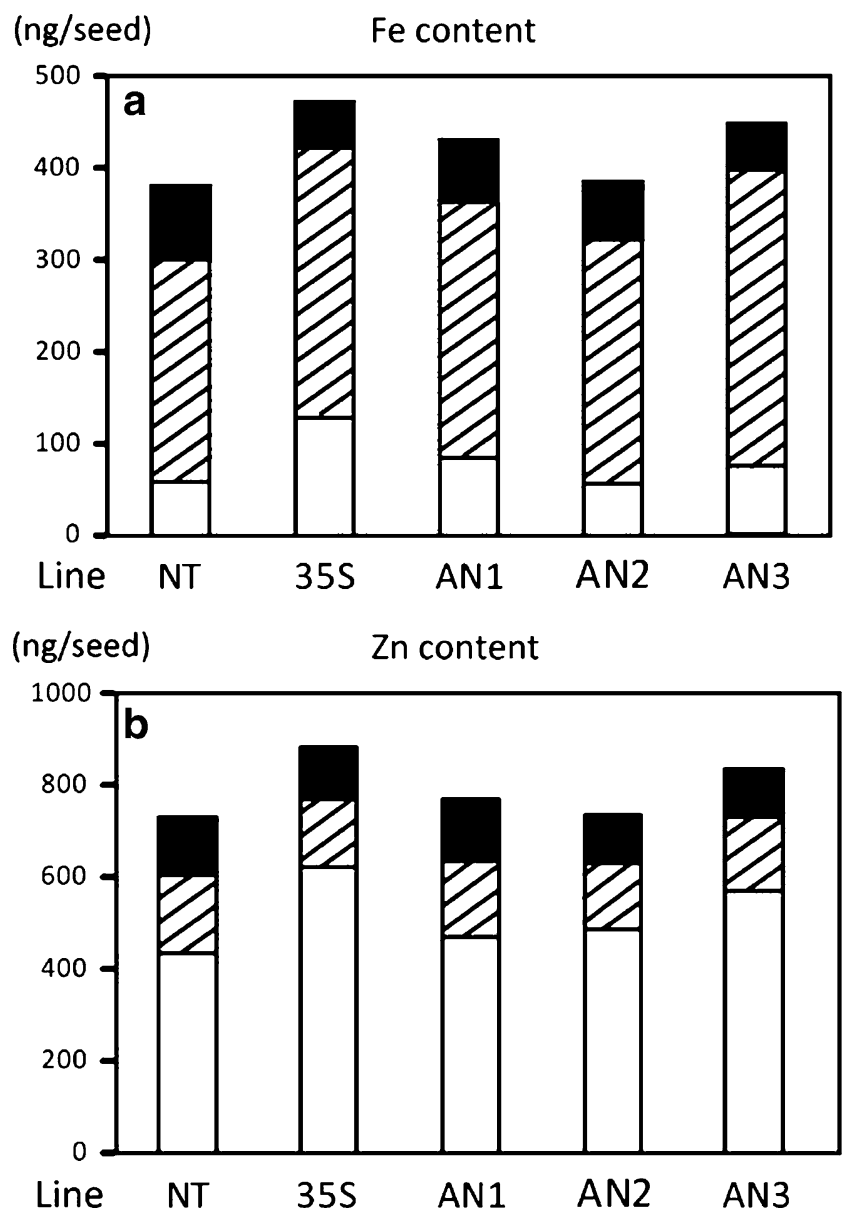

Fig. $5 \mathrm{Fe}$ and $\mathrm{Zn}$ contents per seed in $\mathrm{T}_{2}$ seeds obtained from plants cultured in small pots fertilized with nutrient solution. Fe and $\mathrm{Zn}$ contents per seed were calculated from the metal concentration data shown in Fig. 4. a Fe content per seed. b Zn content per seed. White bar, polished seed parts (endosperm); hatched bar, bran and embryo; black bar, hulls. respectively, compared to the NT line (Fig. 6a). DMA concentrations in $\mathrm{T}_{2}$ seeds of $35 \mathrm{~S}, \mathrm{AN} 1$, and $\mathrm{AN} 3$ lines also increased 4.8-, 2.8-, and 1.7-fold, respectively, compared to the NT line (Fig. 6a). The NA concentration in seeds was correlated with $\mathrm{Fe}$ or $\mathrm{Zn}$ concentration in polished rice seeds in each line (Fig. 6b, c). A correlation between DMA concentration in seeds and $\mathrm{Fe}$ or $\mathrm{Zn}$ concentration in polished seeds in each line was also observed (Fig. 6d, e).

Fe and $\mathrm{Zn}$ concentration and localization in $\mathrm{T}_{2}$ seeds of soil culture

In the second experiment, we cultivated six $T_{1}$ sublines from AN1, AN2, and AN3 in soil culture with slow-release fertilizer to obtain $\mathrm{T}_{2}$ seeds and then measured $\mathrm{Fe}$ and $\mathrm{Zn}$ contents in seed and leaves (Fig. 7). In polished $\mathrm{T}_{2}$ seeds collected from sublines AN1-1, AN3-2, and AN3-6, Fe concentrations increased up to 3.4-, 2.5-, and 2.8-fold, respectively, compared to NT plants (Fig. 7a). AN1-2, AN1-5, AN2-6, AN3-1, and AN3-4 also produced polished $\mathrm{T}_{2}$ seeds with up to 1.5 -fold increases in Fe concentration compared to NT plants. In the remaining sublines, Fe concentrations in polished seeds showed only slight increases, or no increase, compared to NT plants (Fig. 7a). $\mathrm{Zn}$ concentrations in $\mathrm{T}_{2}$ polished rice seeds collected from the AN1-1, AN1-4, AN 3-2, and AN 3-6 sublines increased up to 2.0-, 1.8-, 1.9-, and 2.3-fold, respectively, compared to NT plants (Fig. 7b). The remaining sublines, except for AN2-3, AN2-4, and AN2-5, also produced $\mathrm{Zn}$-enriched polished seeds, with increases of approximately 1.3-1.7 times that observed in NT plants (Fig. 7b). Fe and $\mathrm{Zn}$ content per seed in $\mathrm{T}_{2}$ polished seeds also increased in AN lines compared to the NT line (Fig. 7c, d). Fe and $\mathrm{Zn}$ concentrations in leaves of $\mathrm{AN}$ lines after seed harvest were not significantly increased compared to NT (Fig. 7e, f).

Next, $T_{2}$ seeds were subjected to Prussian blue staining to observe the Fe localization in seeds (Fig. 8). Histochemical analysis showed that Fe was localized mostly in bran and embryo in NT seeds (Fig. 8a). On the other hand, more Fe was localized in endosperm of AN1-1 and AN3-2 line seeds compared to that of NT (Fig. 8b, c).

\section{Discussion}

In rice, NA is thought to function as an $\mathrm{Fe}(\mathrm{II})$ chelator in phloem and to participate in long-distance $\mathrm{Fe}$ transport within the plant (Inoue et al. 2003; Koike et al. 2004). We cultivated transgenic rice lines carrying OsActin1promoter$H v N A S 1$ (AN lines) and 35S promoter-HvNAS1 (35S line), which overexpress the HvNAS1 transgene (Fig. 2). In the $\mathrm{AN}$ lines, Fe and $\mathrm{Zn}$ concentrations increased in polished 

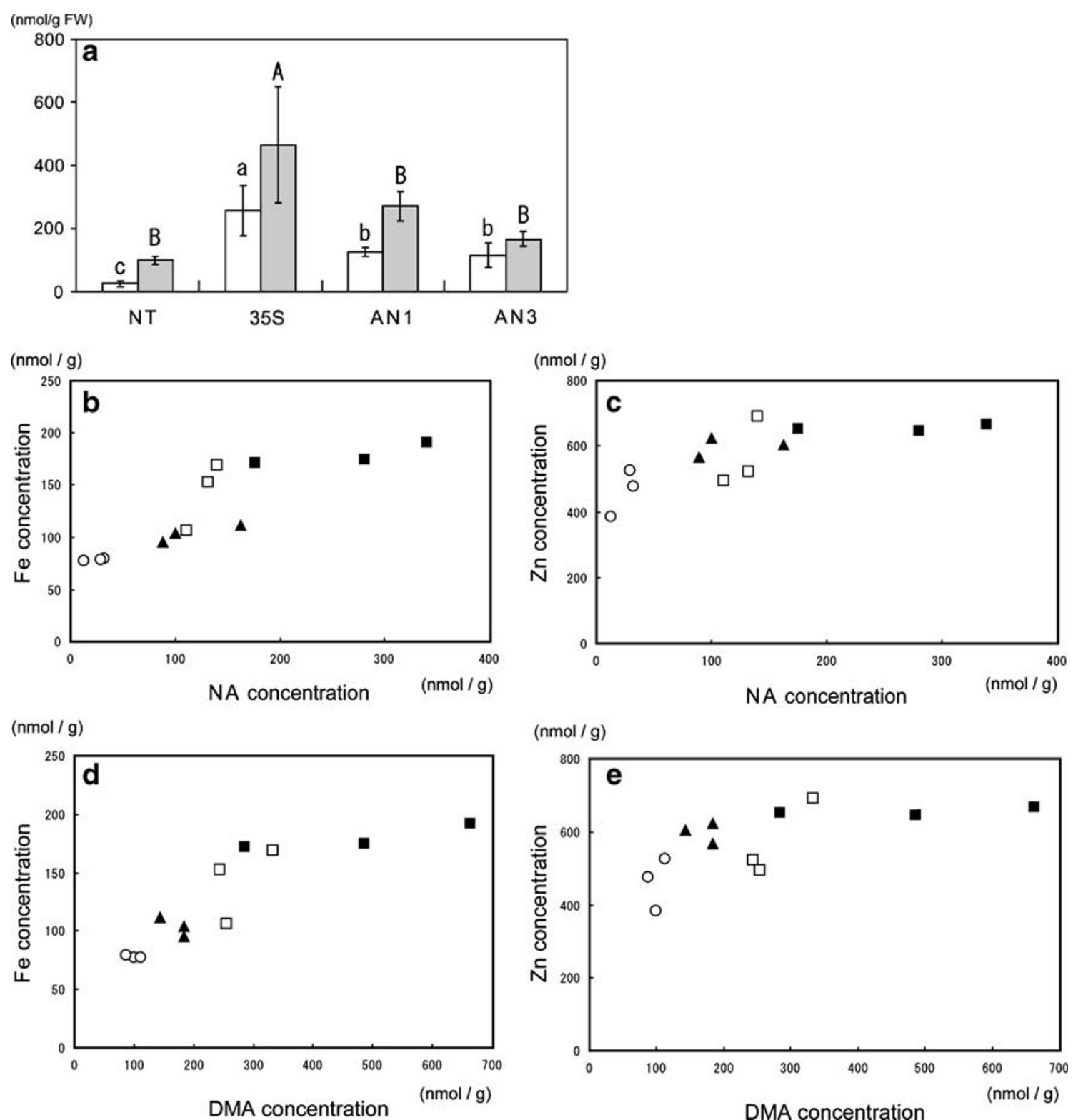

Fig. 6 a NA and DMA concentration in $T_{2}$ seeds obtained from plants cultured in small pots fertilized with nutrient solution. White bars, NA; gray bars, DMA. Distinct letters above the bars indicate significant differences $(p<0.05)$. b-e Scatter diagram between NA or DMA concentration in $\mathrm{T}_{2}$ seeds and $\mathrm{Fe}$ or $\mathrm{Zn}$ concentration in

polished $T_{2}$ seeds for each plant cultured in small pots fertilized with nutrient solution. b NA and Fe concentrations. c NA and $\mathrm{Zn}$ concentrations. d DMA and Fe concentrations. e DMA and $\mathrm{Zn}$ concentrations. White circle, NT lines; black square, 35S lines; white square, AN1 lines; black triangle, AN3 lines.

$\mathrm{T}_{1}$ and $\mathrm{T}_{2}$ seeds cultivated via two distinct methods (Figs. 1, 4, and 7). We observed a positive correlation between the expression of HvNAS1 in the shoots and $\mathrm{Fe}$ and $\mathrm{Zn}$ concentrations in polished and brown rice seeds (Figs. 2 and 4). In addition, a positive correlation was observed between $\mathrm{Fe}$ or $\mathrm{Zn}$ concentration and NA or DMA concentration in seeds (Fig. 6). These results suggest that enhanced NA production increases the translocation of $\mathrm{Fe}$ and $\mathrm{Zn}$ into seeds. Bashir et al. (2006) suggested that DMA-Fe is transported from the roots to shoots in

Fe-sufficient rice plants. In Zn-deficient rice plants, Suzuki et al. (2008b) showed that DMA promotes increased $\mathrm{Zn}$ translocation. We observed increased DMA concentrations in the 35S and AN1 lines (Fig. 3b), which may also have contributed to increased $\mathrm{Fe}$ and $\mathrm{Zn}$ concentrations in these plants.

NA concentration in $T_{2}$ seeds of the $35 \mathrm{~S}$ line and $\mathrm{AN}$ lines also increased compared to the NT line (Fig. 6). Usuda et al. (2009) produced transgenic rice that expressed HvNAS1 under the control of an endosperm-specific 
promoter, $O s G l u B-1$. NA concentration in brown seeds of this transgenic line increased threefold compared to the NT line, and Fe concentration in polished seeds also increased 1.5-fold (data not shown). Higher NA productivity in the endosperm of this transgenic rice might have improved $\mathrm{Fe}$ accumulation in seeds. Thus, enhanced NA synthesis in the $35 \mathrm{~S}$ and AN lines may have improved not only $\mathrm{Fe}$ translocation to polished seeds but also Fe accumulation in polished seeds.

In contrast to polished and brown rice seeds, $\mathrm{Fe}$ and $\mathrm{Zn}$ contents decreased in hulls per seed (Fig. 4). The vascular bundle inside a seed is divided and runs in two directions: one that reaches the ovary, which develops into the bran and endosperm, and another that reaches the hull in a rachilla (Takane and Hoshikawa 1993). NA-Fe(II) and NA-Zn(II) may be more easily transported into the bran and endosperm than into the hull.

Cheng et al. (2007) produced a rice mutant with increased endogenous NA concentrations via knockout of the OsNAAT1 gene, which is the sole Fe deficiency-induced $N A A T$ gene in rice (Inoue et al. 2008). The OsNAAT1 knockout mutant accumulated greater amounts of $\mathrm{Fe}$, but not $\mathrm{Zn}$, in polished rice seeds when cultivated under waterlogged conditions. Because the mutant expressed a wide range of $\mathrm{Fe}$ deficiency-responsive genes, the OsNAAT1 knockout mutant appears to induce physiological Fe deficiency. In contrast, we found that the overexpression of the NAS gene alone is sufficient to increase $\mathrm{Fe}$ and $\mathrm{Zn}$ concentrations in seeds.

Higuchi et al. (2001a, b) produced rice lines expressing a genomic fragment of $H v N A S 1$, resulting in increased NA content in the roots and leaves under Fe-sufficient conditions. However, $\mathrm{Fe}$ and $\mathrm{Zn}$ concentrations did not increase in seeds collected from $H v N A S 1$ genome insertion rice plants when grown under $\mathrm{Fe}$-deficient conditions in calcareous paddy fields or under Fe-sufficient conditions in andosol paddy fields (Masuda et al. 2008; Suzuki et al. 2008a). This difference in Fe accumulation in rice seeds is thought to be related to the expression of the NAS transgene and subsequent NA content. Under Fe-sufficient conditions, NA concentrations in $35 S-H v N A S 1$ lines were higher compared to HvNAS1 genome insertion lines (Higuchi et al. 2001a). Moreover, in 35S-HvNAS1 lines, the NAS transgene is presumably expressed throughout the rice plant, which would promote widespread Fe circulation and bioavailability.

In addition to seed $\mathrm{Fe}$ and $\mathrm{Zn}$ concentration, $\mathrm{Fe}$ and $\mathrm{Zn}$ content per seed in $T_{2}$ seeds of $A N$ lines also increased compared to those of the NT line (Fig. 5). There was no significant correlation between yield and Fe or Zn concentration in polished seeds in each $\mathrm{T}_{2}$ plant of NT, AN1, and AN3 lines (data not shown). Therefore, it can be postulated that increased $\mathrm{Fe}$ and $\mathrm{Zn}$ concentration in seeds of transgenic lines was not due to decreased grain size or yield. Fe and $\mathrm{Zn}$ concentration in leaves of AN lines were not significantly increased compared to NT (Fig. 7e, f). Overexpression of $H v N A S 1$ might not lead to $\mathrm{Fe}$ and $\mathrm{Zn}$ overaccumulation in leaves. We observed no symptom of Fe toxicity in transgenic rice lines.

Actin promoter is reported to induce generally stronger expression than $35 \mathrm{~S}$ promoter when introduced into graminaceous plants such as rice (McElroy et al. 1990). Therefore, we produced not only $35 \mathrm{~S}$ lines but also AN lines in order to obtain new lines with higher $\mathrm{Fe}$ and $\mathrm{Zn}$ concentrations. However, 35S line accumulated more NA, DMA than AN lines (Fig. 3). Fe and Zn concentration in seeds were also higher in $35 \mathrm{~S}$ line than AN lines (Fig. 4). Nevertheless, AN lines also showed higher $\mathrm{Fe}$ and $\mathrm{Zn}$ concentrations in seeds than NT line.

In lines AN1, AN2, and AN3, Fe accumulation was relatively low in $T_{2}$ polished rice seeds compared to $T_{1}$ seeds (Figs. 1, 4, and 7). However, marked increases in Fe and $\mathrm{Zn}$ concentrations, similar to $\mathrm{T}_{1}$ seeds, were achieved in some $T_{2}$ sublines (Fig. 7). These results may reflect differences in the genotype of each subline. Fe concentrations in the $35 \mathrm{~S}$ line were consistently high because of its stable homozygous genotype. Further selection of AN sublines with high $\mathrm{Fe}$ and $\mathrm{Zn}$ content in $\mathrm{T}_{2}$ seeds such as AN1-1 and AN3-6 would produce stable increases in micronutrient content.

Our results clarified the effect of overexpression of $N A S$ to increase $\mathrm{Fe}$ and $\mathrm{Zn}$ concentrations in rice seeds. In previous studies, we generated a variety of transgenic rice lines bearing one or more of the genes involved in MA biosynthesis (Suzuki et al. 2008a). Among these, a transgenic rice line carrying a barley genomic fragment containing the IDS3 gene showed increased $\mathrm{Fe}$ and $\mathrm{Zn}$ contents when grown in calcareous or andosol paddy fields (Masuda et al. 2008; Suzuki et al. 2008a). Therefore, we assume that introduction of barley IDS3 genome fragments into rice also has high potential to increase Fe concentration in rice seeds. Vasconcelos et al. (2003) produced transgenic rice plants with introduced Ferritin genes into high $\mathrm{Fe}$ variety IR68144, which resulted in increase of both Fe and $\mathrm{Zn}$ concentration in polished rice up to 3.7 or 1.4 times. Wirth et al. (2009) also reported that constitutive overexpression of AtNAS1 in combination with endospermspecific expression of Ferritin is an effective method to increase $\mathrm{Fe}$ concentration in polished rice seeds. Because the IDS3 genome fragment might enhance $\mathrm{Fe}$ and $\mathrm{Zn}$ uptake from the soil, concomitant $N A S$ overexpression might also increase $\mathrm{Fe}$ and $\mathrm{Zn}$ transport to the seeds, while endosperm-specific expression of Ferritin might further enhance Fe storage ability in seeds. Thus, we postulate that combination of these three genes may allow further improvement in $\mathrm{Fe}$ and $\mathrm{Zn}$ accumulation in seeds. 

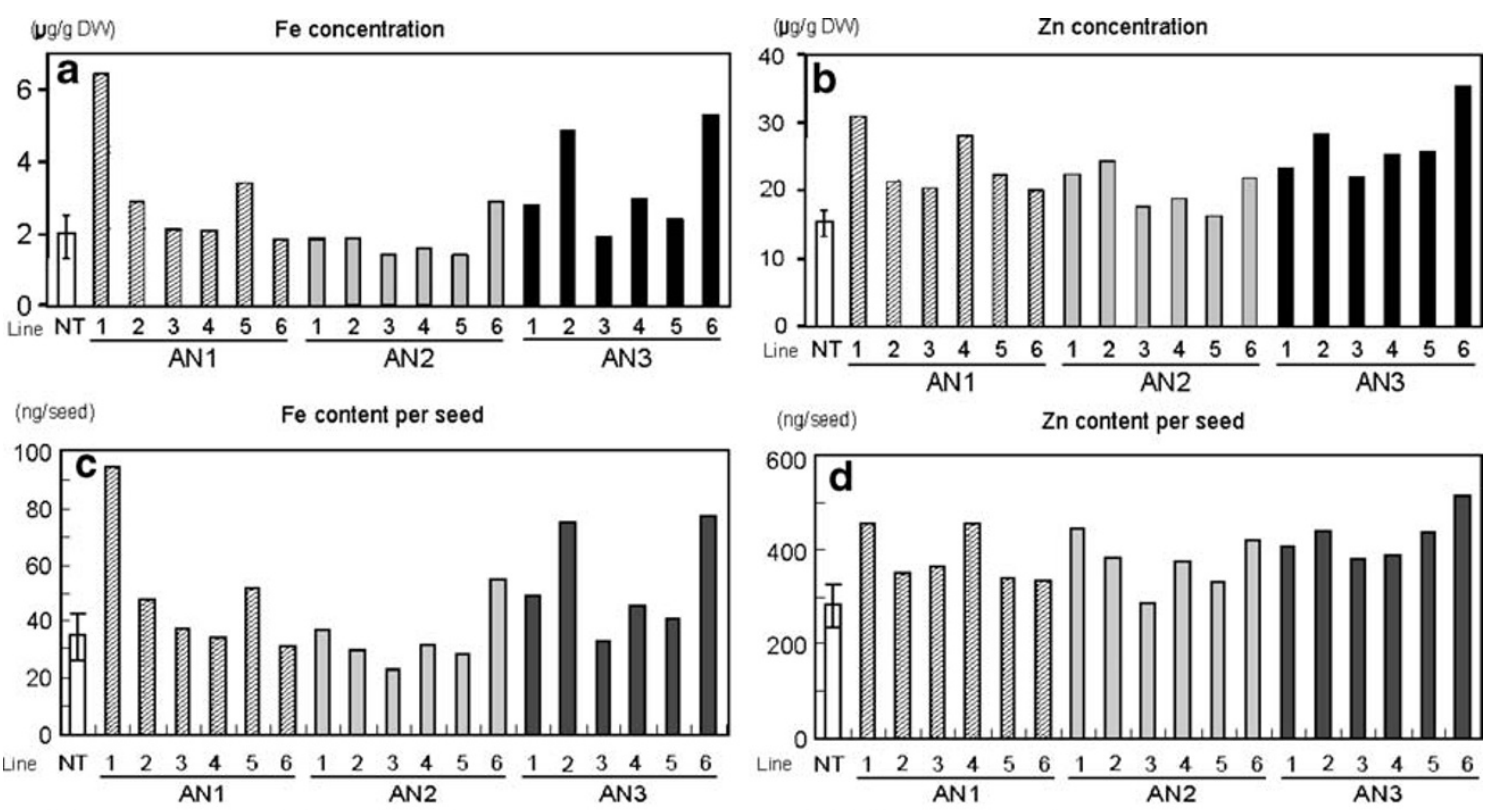

(ng/seed) Zn content per seed
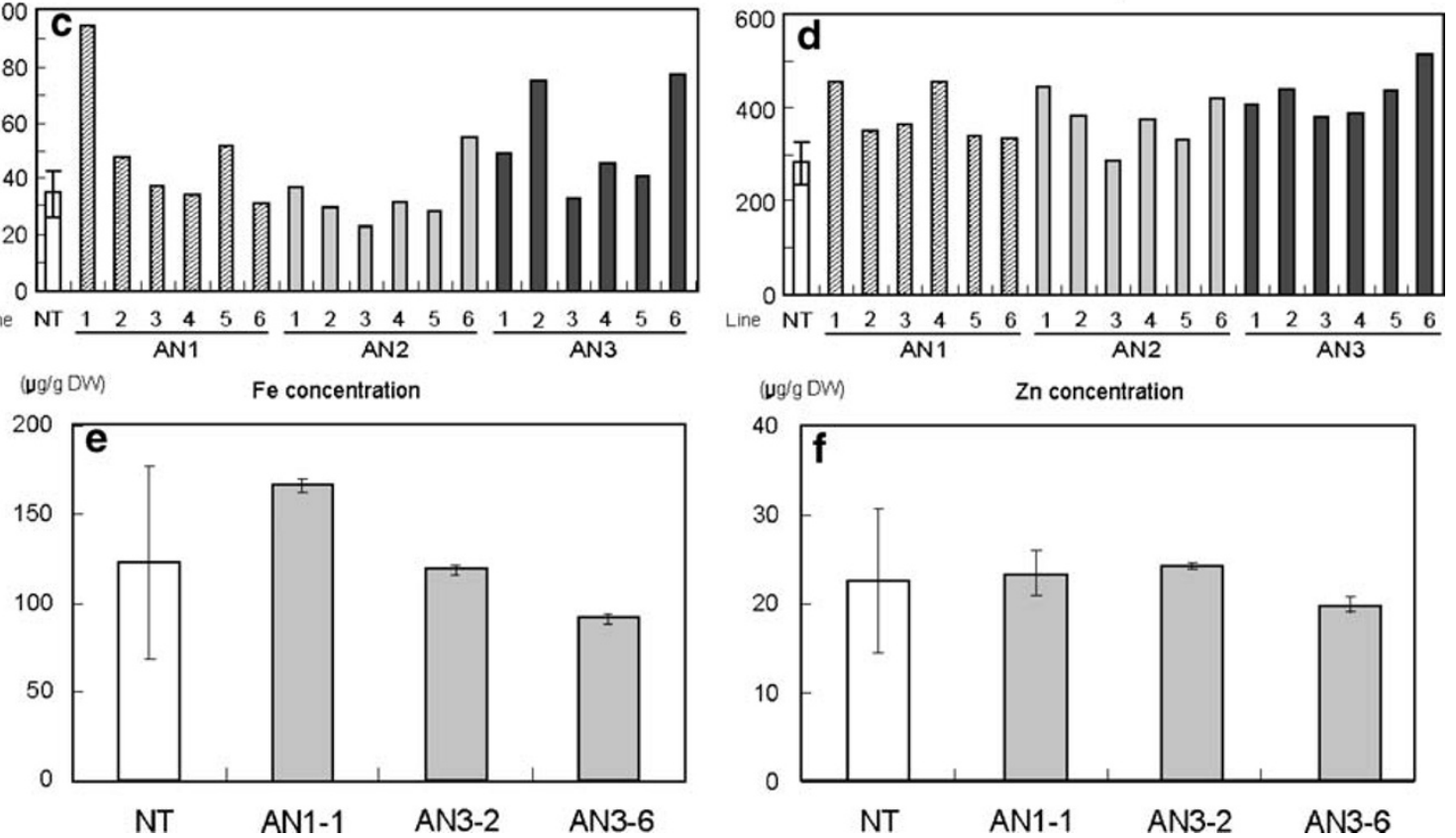

Fig. $7 \mathrm{Fe}$ and $\mathrm{Zn}$ concentrations in polished $\mathrm{T}_{2}$ seeds and leaves obtained from soil culture with slow-release fertilizer. Ten wellpolished seeds obtained from the main tillers of six plants in each of six $T_{1}$ sublines were analyzed. a Fe concentration. b $\mathrm{Zn}$ concentration. c Fe contents per seed. d $\mathrm{Zn}$ contents per seed. The data shown for the NT line represent the mean \pm standard error for six plants cultivated in

\section{Methods}

Plant materials

Japonica rice (Oryza sativa L.), cultivar Tsukinohikari, was used as the NT (non-transgenic) control and for transformation. The transgenic rice line bearing $35 S-H v N A S 1$ (35S) was originally produced by Higuchi et al. (2001a) and designated S11. This S11 line was cultured through several generations to select a homozygous line, which was then used in all analyses.

Construction of OsActin1-HvNAS1 and rice transformation

The OsActin1-HvNAS1-nopaline synthase gene 3'-UTR (tNOS) cassette was constructed as follows. The OsActin1

promoter was amplified using rice genomic DNA and the primers 5'-AAG CTT CGA GGT CAT TCA TAT GCT TG-3' and 5'-GTC GAC CTT CTA CCT ACA AAA AAG CT-3' and then subcloned using the HindIII and SalI sites in the pUC18 vector (TaKaRa Bio, Shiga, Japan). This promoter sequence was excised from the vector using HindIII and $X b a \mathrm{I}$ and then ligated into pBluescript $\mathrm{SK}(+)$ (Stratagene, La Jolla, CA, USA). The HvNASI open reading frame (ORF) -tNOS sequence was amplified via polymerase chain reaction using the 35S-HvNAS1-tNOS cassette (Higuchi et al. 2001a) as a template and the primers 5'-GCA CTA GTA GCC ATG GAT GCC CAG AAC AGG AGG-3' and 5'-GCA CTA GTA TTC CCG ATC TAG TAA CAT AGA TG-3', both of which contained a SpeI site at the $5^{\prime}$-end. After sequence verification, the HvNAS1-tNOS fragment was digested with SpeI, whereas the OsActinl promoter in the $\mathrm{SK}(+)$ vector was 
Fig. $8 \mathrm{Fe}$ localization of $\mathrm{T}_{2}$ seeds obtained from soil culture. Brown seeds were cut and stained by prussian blue staining. a NT. b AN1-1. c AN3-2. d AN3-6.
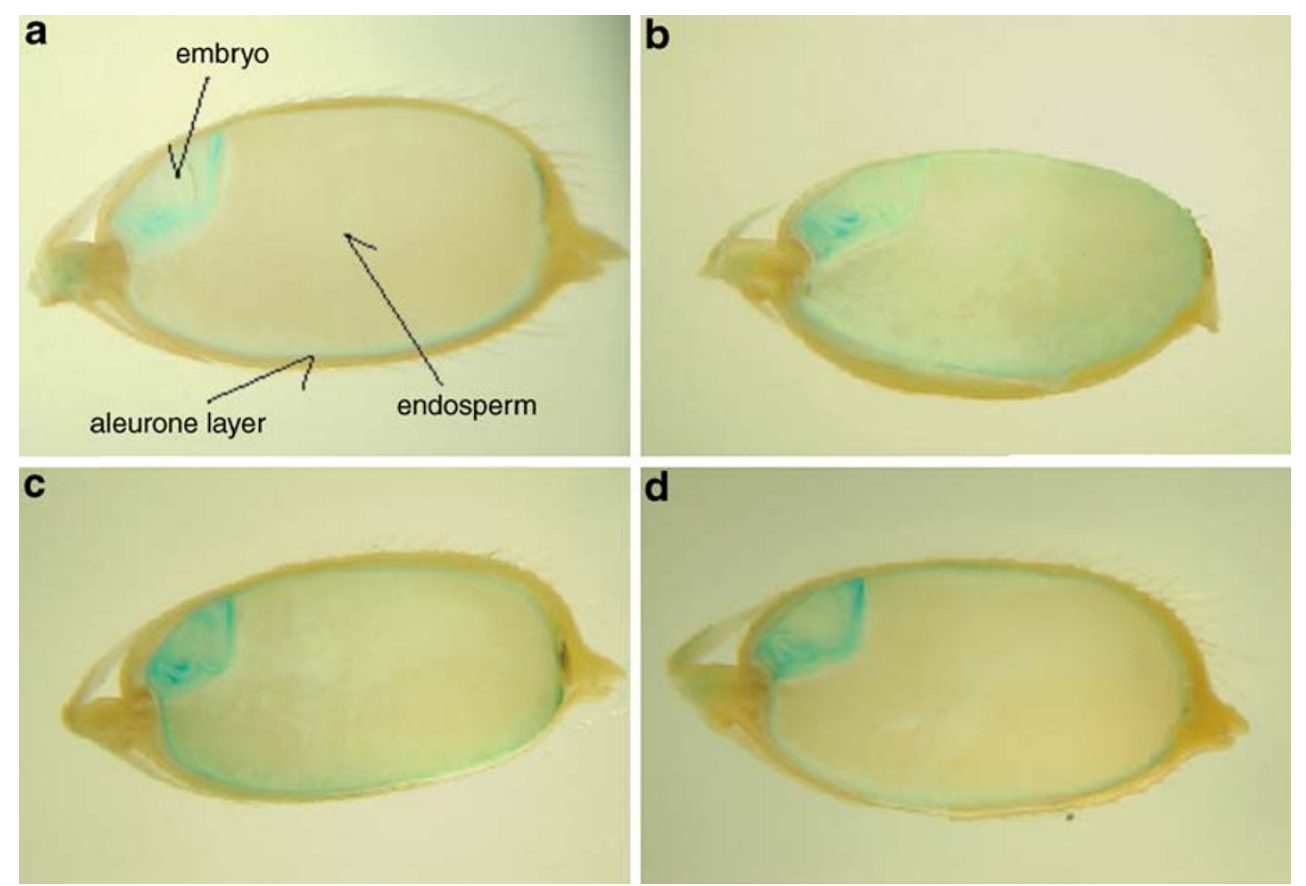

digested with $X b a \mathrm{I}$. These two fragments were then ligated together to produce the OsActin1-HvNAS1-tNOS cassette. To create transgenic lines, this cassette was excised using NotI and $K p n I$ and then cloned into the same sites in the pBIGRZ1 vector (Akiyama et al. 1997). Agrobacterium tumefaciens (C58) was used to carry this construct into O. sativa L. cv. Tsukinohikari using the method developed by Hiei et al. (1994) and modified by Akiyama et al. (1997). Twenty OsActin1-HvNAS1-tNOS transgenic (AN) lines were obtained as $\mathrm{T}_{0}$ transgenic plants.

\section{Plant growth conditions}

Twenty $\mathrm{T}_{0}$ AN lines carrying OsActin 1-HvNAS1 and control NT plants were cultivated in $3.5-\mathrm{CL}$ pots $(1,000-\mathrm{ml}$ volume; Kaneya, Aichi, Japan) containing artificial soil composed of a 2:1 mixture of bonsol-ichigou (Sumitomo Kagaku, Tokyo, Japan) and vermiculite (Green Tec, Tochigi, Japan). The soil was evenly fertilized with $3.5 \mathrm{~g}$ of a slow-release fertilizer, LongTotal-70 (Chisso-Asahi, Tokyo, Japan; N/P/K, 13:11:13), and $3.5 \mathrm{~g}$ of Long Total-140 (Chisso-Asahi; $\mathrm{N} / \mathrm{P} / \mathrm{K}, 13: 11: 13$ ) per plant. Plants were grown in a greenhouse under natural light conditions, with $14 \mathrm{~h}$ of light at $30{ }^{\circ} \mathrm{C}$ and $10 \mathrm{~h}$ of dark at $25^{\circ} \mathrm{C}$. Plants were transplanted on 18 December 2006 and seeds harvested on 4 June 2007. Twelve lines were subjected to metal concentration analysis.

$\mathrm{NT}, \mathrm{T}_{1} \mathrm{AN}$, and $35 \mathrm{~S}$ seeds were germinated on Murashige and Skoog (MS) medium. After 3 weeks, six seedlings from each line were transferred into nutrient solution $\left[2 \mathrm{mM} \mathrm{Ca}\left(\mathrm{NO}_{3}\right)_{2}, 0.5 \mathrm{mM} \mathrm{MgSO}_{4}, 0.7 \mathrm{mM}\right.$ $\mathrm{K}_{2} \mathrm{SO}_{4}, 0.1 \mathrm{mM} \mathrm{KCl}, 0.1 \mathrm{mM} \mathrm{KH} \mathrm{PO}_{4}, 0.1 \mathrm{mM} \mathrm{Fe(III)-}$

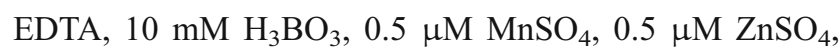
$0.2 \mu \mathrm{M} \mathrm{CuSO}_{4}$, and $\left.0.01 \mu \mathrm{M}\left(\mathrm{NH}_{4}\right)_{6} \mathrm{Mo}_{7} \mathrm{O}_{25}\right]$ and grown in a greenhouse under the conditions described above. The $\mathrm{pH}$ of the culture solution was adjusted daily to between 5.5 and 5.8 with $1 \mathrm{~N} \mathrm{HCl}$. Twenty-three days after transplanting, leaves and roots were harvested for Northern blot analysis and the determination of NA and DMA concentrations.

For the metal concentration analysis, $\mathrm{T}_{1}$ transgenic and NT lines were grown using two different methods. In the first method, three lines of $\mathrm{T}_{1}$ AN seeds (AN1, AN2, and AN3), 35S line seeds, and NT line seeds were germinated on MS medium for 3 weeks, and then seedlings were transplanted into small ceramic pots (250-ml volume) filled with bonsol-ichigou, but no additional fertilizer. Six pots containing three seedlings each were prepared for each line, and each pot was submerged in nutrient solution. The plants were settled on 6 August 2007 and harvested on 28 November 2007. $T_{2}$ seeds were used in the metal concentration analysis.

In the second method, NT seeds and $\mathrm{T}_{1}$ seeds from lines AN1, AN2, and AN3 were germinated on MS medium on 17 August 2007. On 3 September 2007, six $T_{1}$ seedlings from each line were settled in 3.5-CL pots containing artificial soil (2:1 mixture of bonsol-ichigou and vermiculite). The soil was fertilized evenly with $3.5 \mathrm{~g}$ of LongTotal-70 and $3.5 \mathrm{~g}$ of Long Total-140 per plant. Plants were grown in a greenhouse under natural light conditions, with $14 \mathrm{~h}$ of light at $30^{\circ} \mathrm{C}$ and $10 \mathrm{~h}$ of dark at $25^{\circ} \mathrm{C}$. The plants were harvested on 5 January 2008 and the seeds used in the metal concentration analysis. 


\section{Northern blotting}

The ORF sequence for HvNAS1 (AB010086) was used to create an HvNAS1 probe (Suzuki et al. 2006). This fragment was labeled with $\left[\mathrm{a}^{3}{ }^{32} \mathrm{P}\right]-\mathrm{dATP}$ using the random labeling method; labeled DNA was purified using a ProbeQuant G-50 micro-column (Pharmacia, Uppsala, Sweden). Total RNA in shoots was extracted using the SDS-phenol method. Total RNA ( $20 \mu \mathrm{g}$ per lane) was separated on $1.4 \%(w / v)$ agarose gels. Blotting, hybridization and radioactive detection, and quantification were performed as previously described (Ogo et al. 2006).

\section{Determination of NA and DMA concentrations}

For determination of endogenous NA and DMA concentrations in the $\mathrm{T}_{1}$ plant body, approximately $5 \mathrm{~g}$ of frozen shoots and roots from each transgenic and NT line was ground and homogenized with a mortar and pestle and then suspended in $100 \mathrm{ml}$ of deionized hot water $\left(80{ }^{\circ} \mathrm{C}\right)$ for $20 \mathrm{~min}$. After suspension, the samples were centrifuged at $8,000 \times g$ for $15 \mathrm{~min}$ and the supernatants collected. These extraction steps were the same for both NA and DMA measurement. For HPLC analysis of NA, the supernatants were subjected to ion-exchange purification, evaporated, and filtered as previously described (Wada et al. 2006). For HPLC analysis of DMA, the supernatants were subjected to ion-exchange purification, evaporated, and filtered under different conditions as previously described (Suzuki et al. 2006). All steps for DMA measurement were performed in the dark. One sample was prepared from each line and analyzed in triplicate to determine the concentrations of NA and DMA.

For determination of NA and DMA concentrations in $\mathrm{T}_{2}$ seeds, ten brown seeds from each of three lines of $35 \mathrm{~S}$, $\mathrm{AN} 1$, and AN3, whose $\mathrm{Fe}$ concentrations in $\mathrm{T}_{2}$ polished seeds were higher than in the other three lines, were selected randomly. These seeds were crushed in a MultiBeads Shocker (Yasui Kikai, Osaka, Japan). Samples were mixed with $400 \mu 1$ of $80 \%$ ethanol, centrifuged at $12,000 \times g$ for $5 \mathrm{~min}$, and the supernatants were collected for measurement. This step was repeated three times. One hundred microliters of supernatant was filtered in a $10 \mathrm{~K}$ Nanosep centrifugal device (Pall, Ann Arbor, MI, USA). One sample was prepared from each line and the concentrations of NA and DMA were analyzed by LC/ESI-TOF-MS (JEOL, Tokyo, Japan) as previously described (Kakei et al. 2007; Wada et al. 2007).

Metal concentration analysis

Brown seeds were collected randomly from the ear of the main tiller (the tiller in the center or the largest among all tillers in one plant). Ten $T_{1}$ seeds from each $T_{0}$ transgenic line or ten $T_{2}$ seeds from each $T_{1}$ transgenic lines were dried overnight at $80{ }^{\circ} \mathrm{C}$ in a heat-dry machine. After determining the dry weight of each sample, the seeds were digested in $1 \mathrm{ml}$ of $13 \mathrm{M} \mathrm{HNO}_{3}$ and $1 \mathrm{ml}$ of $8.8 \mathrm{M} \mathrm{H}_{2} \mathrm{O}_{2}$ (Wako, Osaka, Japan) at $200{ }^{\circ} \mathrm{C}$ for $20 \mathrm{~min}$ with MARS Xpress (CEM, Matthews, NC, USA). After digestion, the samples were diluted to a volume of $5 \mathrm{ml}$ and analyzed via inductively coupled plasma atomic emission spectrometry (SPS1200VR; Seiko, Tokyo, Japan).

To polish seeds, 30 brown seeds from the ear of the main tiller were placed into a 2-ml tube and shaken vigorously for $150 \mathrm{~s}$ at 2,500 rpm for at least four cycles using a Multi-Beads Shocker (Yasui Kikai). Ten well-polished seeds from each plant were selected. After removing the embryos, polished seeds were dried overnight, weighed, digested, and then analyzed as described above.

Rice bran (approximately $30-50 \mathrm{mg}$ ) was obtained as a by-product of the polishing process. Rice bran was dried overnight, weighed, and then digested at $220{ }^{\circ} \mathrm{C}$ for $20 \mathrm{~min}$ in $1 \mathrm{ml}$ of $13 \mathrm{M} \mathrm{HNO}_{3}$ and $1 \mathrm{ml}$ of $8.8 \mathrm{M} \mathrm{H}_{2} \mathrm{O}_{2}$. Metal analysis was performed as described above. Rice hulls (approximately $80-160 \mathrm{mg}$ ) were also dried overnight, weighed, digested, and subjected to metal analysis as described above. Leaf sheaths and leaf blades were harvested, dried, and crashed by homogenizer (Fujiwara Seisakusyo, Tokyo, Japan). About $200 \mathrm{mg}$ of crashed leaves was then digested and subjected to metal analysis as described above.

Fe localization by prussian blue staining

$\mathrm{T}_{2}$ brown seeds were soaked in water overnight and were cut by a razor. Then, the samples were soaked in $2 \% \mathrm{HCl}$ (Wako) and $2 \%$ potassium hexacyanoferrate(II) trihydrate (Wako) for $1.5 \mathrm{~h}$. Stained samples were washed with distilled water and observed by stereoscopic microscope (AX10 canHRP, ZEISS).

\section{Statistics}

Analysis of variance with the Student-Newman-Keuls test was used to compare data. The level of significance was set at $p<0.05$. The statistical software package JMP7 (SAS Institute, Cary, NC, USA) was used in all analyses.

Acknowledgments We thank Mr. T. Maruyama for cultivating rice in the greenhouse. We also thank Ms. R.N. Itai for technical advice regarding Northern blot analysis.

Open Access This article is distributed under the terms of the Creative Commons Attribution Noncommercial License which permits any noncommercial use, distribution, and reproduction in any medium, provided the original author(s) and source are credited. 


\section{Reference}

Akiyama K, Nakamura S, Suzuki T, Wisniewska I, Sasaki N, Kawasaki S. Development of a system of rice transformation with long genome inserts for their functional analysis for positional cloning. Plant Cell Physiol. 1997;38:s94.

Bashir K, Inoue H, Nagasaka S, Takahashi M, Nakanishi H, Mori S, et al. Cloning and characterization of deoxymugineic acid synthase genes from graminaceous plants. J Biol Chem. 2006;281:32395-402.

Cheng L, Wang F, Shou H, Huang F, Zheng L, He F, et al. Mutation in nicotianamine aminotransferase stimulated the $\mathrm{Fe}(\mathrm{II})$ acquisition system and led to iron accumulation in rice. Plant Physiol. 2007;145:1647-57.

Curie C, Panaviene Z, Loulergue C, Dellaporta SL, Briat JF, Walker EL. Maize yellow stripe1 encodes a membrane protein directly involved in Fe(III) uptake. Nature. 2001;409:346-9.

Goto F, Yoshihara T, Shigemoto N, Toki S, Takaiwa F. Iron fortification of rice seed by the soybean ferritin gene. Nat Biotechnol. 1999;17:282-6.

Hell R, Stephan UW. Iron uptake, trafficking and homeostasis in plants. Planta. 2003;216:541-51.

Hiei Y, Ohta S, Komari T, Kumashiro T. Efficient transformation of rice (Oryza sativa L.) mediated by Agrobacterium and sequence analysis of the boundaries of the T-DNA. Plant J. 1994;6: 271-82.

Higuchi K, Kanazawa K, Nishizawa NK, Chino M, Mori S. Purification and characterization of nicotianamine synthase from Fe-deficient barley roots. Plant Soil. 1994;165: $173-9$.

Higuchi K, Takahashi M, Nakanishi H, Kawasaki S, Nishizawa NK, Mori S. Analysis of transgenic rice containing barley nicotianamine synthase gene. Soil Sci Plant Nutr. 2001a;47: 315-22.

Higuchi K, Watanabe S, Takahashi M, Kawasaki S, Nakanishi H, Nishizawa NK, et al. Nicotianamine synthase gene expression differs in barley and rice under Fe-deficient conditions. Plant J. 2001b;25:159-67.

Inoue $\mathrm{H}$, Higuchi K, Takahashi M, Nakanishi H, Mori S, Nishizawa NK. Three rice nicotianamine synthase genes, OsNAS1, OsNAS2, and OsNAS3 are expressed in cells involved in long-distance transport of iron and differentially regulated by iron. Plant J. 2003;36:366-81.

Inoue H, Takahashi M, Kobayashi T, Suzuki M, Nakanishi H, Mori S, et al. Identification and localisation of the rice nicotianamine aminotransferase gene OsNAAT1 expression suggests the site of phytosiderophore synthesis in rice. Plant Mol Biol. 2008;66: 193-203.

Kakei Y, Wada Y, Yamaguchi I, Takahashi M, Nakanishi H, Mori S, et al. A new sensitive quantitative method for $2^{\prime}$-deoxymugineic acid and nicotianamine using LC/ESI-TOF-MS. Plant Cell Physiol. 2007;48:S43.

Klatte M, Schuler M, Wirtz M, Fink-Straube C, Hell R, Bauer P. The analysis of arabidopsis nicotianamine synthase mutants reveals functions for nicotianamine in seed iron loading and iron deficiency responses. Plant Physiol. 2009;150:257-71.

Kobayashi T, Nishizawa NK. IV 3. Regulation of iron and zinc uptake and translocation in rice. In: Hirano HY, Hirai A, Sano Y, Sasaki T, editors. Biotechnology in Agriculture and Forestry 62 Rice Biology in the Genomics Era. Berlin: Springer; 2008. p. $321-35$

Koike S, Inoue H, Mizuno D, Takahashi M, Nakanishi H, Mori S, et al. OsYSL2 is a rice metal-nicotianamine transporter that is regulated by iron and expressed in the phloem. Plant J. 2004; $39: 415-24$
Lucca P, Poletti S, Sautter C. Genetic engineering approaches to enrich rice with iron and vitamin A. Physiol Plant. 2006; 126:291-303.

Masuda H, Suzuki M, Morikawa KC, Kobayashi T, Nakanishi H, Takahashi $\mathrm{M}$, et al. Increase in iron and zinc concentrations in rice grains via the introduction of barley genes involved in phytosiderophore synthesis. Rice. 2008;1:100-8.

McElroy D, Zhang W, Cao J, Wu R. Isolation of an efficient actin promoter for use in rice transformation. Plant Cell. 1990;2:16371.

Mihashi S, Mori S. Characterization of mugineic acid-Fe transporter in Fe-deficient barley roots using the multicompartment transport box method. Biol Met. 1989;2:164-54.

Ogo Y, Itai RN, Nakanishi H, Inoue H, Kobayashi T, Suzuki M, et al. Isolation and characterization of IRO2, a novel iron-regulated bHLH transcription factor in graminaceous plants. J Exp Bot. 2006;57:2867-78

Pich A, Scholz G. Translocation of copper and other micronutrients in tomato plants (Lycopersicon esculentum Mill.): nicotianaminestimulated copper transport in the xylem. J Exp Bot. 1996;294:41-7.

Qu LQ, Yoshihara T, Ooyama A, Goto F, Takaiwa F. Iron accumulation does not parallel the high expression level of ferritin in transgenic rice seeds. Planta. 2005;222:225-33.

Rudolph A, Becker R, Scholz G, Procházka Z, Toman J, Macek T, et al. The occurrence of the amino acid nicotianamine in plants and microorganisms: a reinvestigation. Biochemie und Physiologie der Pflanzen. 1985; 180:557-63.

Sandstead HH. Zinc deficiency: a public health problem? Am J Dis Child. 1991;145:853-9.

Stephan UW, Schmidke I, Stephan VW, Scholz G. The nicotianamine molecule is made-to-measure for complexation of metal micronutrients in plants. Biometals. 1996;9:84-90.

Stoltzfus RJ, Dreyfuss ML. Guidelines for the use of iron supplements to prevent and treat iron deficiency anemia. Washington: ILSI Press; 1998.

Suzuki M, Takahashi M, Tsukamoto T, Watanabe S, Matsuhashi S, Yazaki $\mathrm{J}$, et al. Biosynthesis and secretion of mugineic acid family phytosiderophores in zinc-deficient barley. Plant J. 2006;48:85-97.

Suzuki M, Morikawa KC, Nakanishi H, Takahashi M, Saigusa M, Mori S, et al. Transgenic rice lines that include barley genes have increased tolerance to low iron availability in a calcareous paddy soil. Soil Sci Plant Nutr. 2008a;54:77-85.

Suzuki M, Tsukamoto $\mathrm{T}$, Inoue $\mathrm{H}$, Watanabe $\mathrm{S}$, Matsuhashi $\mathrm{S}$, Takahashi M, et al. Deoxymugineic acid increases $\mathrm{Zn}$ translocation in Zn-deficient rice plants. Plant Mol Biol. 2008b;66:609-17.

Takagi S. Naturally occurring iron-chelating compounds in oat- and rice-root washings. Soil Sci Plant Nutr. 1976;22:423-33.

Takahashi M, Terada Y, Nakai I, Nakanishi H, Yoshimura E, Mori S, et al. Role of nicotianamine in the intracellular delivery of metals and plant reproductive development. Plant Cell. 2003;15:126380.

Takane M, Hoshikawa K. Science of the rice plant, volume one, morphology. Tokyo: Nobunkyo Press; 1993. p. 325-6.

Usuda K, Wada Y, Ishimaru Y, Kobayashi T, Takahashi M, Nakanishi H, et al. Genetically engineered rice containing larger amounts of nicotianamine to enhance the antihypertensive effect. Plant Biotechnol J. 2009;7:87-95.

Vasconcelos M, Datta K, Oliva N, Khalekuzzaman M, Torrizo L, Krishnan S, et al. Enhanced iron and zinc accumulation in transgenic rice with the ferritin gene. Plant Sci. 2003;164: 371-8.

Wada Y, Kobayashi T, Takahashi M, Nakanishi H, Mori S, Nishizawa NK. Metabolic engineering of Saccharomyces cerevisiae producing nicotianamine: potential for industrial 
biosynthesis of a novel antihypertensive substrate. Biosci Biotechnol Biochem. 2006;70:1408-15.

Wada Y, Yamaguchi I, Takahashi M, Nakanishi H, Mori S, Nishizawa NK.

Highly sensitive quantitative analysis of nicotianamine using LC/ ESI-TOF-MS with an internal standard. Biosci Biotechnol Biochem. 2007;71:435-41.
Wirth J, Poletti S, Aeschlimann B, Yakandawala N, Drosse B, Osorio S, et al. Rice endosperm iron biofortification by targeted and synergistic action of nicotianamine synthase and ferritin. Plant Biotechnol J. 2009;7:1-14.

World Health Organization (WHO). World health report reducing risks, promoting healthy life. Geneva: WHO; 2002. 